\title{
Variation in the branching pattern of coeliac trunk-Case report
}

\author{
Suresh $\mathrm{T}^{1}$, Sangeeta $\mathrm{M}^{2}$ \\ ${ }^{1,2}$ Department of Anatomy,Sri Devaraj Urs Medical College,Kolar, Karnataka.
}

\begin{abstract}
The Coeliac trunk is the first Ventral branch of abdominal aorta arising at the level of twelfth thoracic $\left(T_{12}\right)$ vertebra. The hepatic, splenic and left gastric arteries are considered as main classic branches of Coeliactrunk. We report a case of variation in the branching pattern of Coeliac trunk wherein the left inferior phrenic artery was seen arising from left gastric artery and there were two accessory hepatic arteries, one of them arising from left gastric artery and another one arising from common hepatic artery distal to the origin of hepatic artery. Cystic artery was seen arising from accessory hepatic artery. The right gastric artery is arising from gastro-duodenal artery. The gallbladder was distended with multiple stones, the wall of the gallbladder was thickened and there were adhesions connecting the gallbladder to liver bed and portahepatis. Knowledge of these vascular anomalies is important in handling patients undergoing diagnostic angiography for gastrointestinal bleeding, Coeliac axis compression syndrome or prior to an operative procedure or transcatheter therapy.
\end{abstract}

Key Words- Accessory hepatic arteries, Coeliac trunk, gallbladder,Left gastric artery, Right gastric artery.

\section{Introduction}

Anomalousblood vessels are always interesting from a scientific point of view since they often shed light on obscure problems of phylogeny and ontogeny. The unusual embryological development of the ventral splanchnic arteries can lead to considerable variations in the branching pattern of Coeliactrunk.

The Coeliac trunk is the first ventral branch of the abdominal aorta and it arises first below the aortic hiatus at level of T12-L1 vertebra. It passes horizontally forwards and divides into left gastric, common hepatic and splenic arteries ${ }^{(1)}$. This trifurcation was first described by Halleri in 1756. This TripusHalleri was and is still being considered to be the normal appearance of the Coeliac trunk ${ }^{(2)}$.

Each dorsal aorta even before the stage of its fusion gives ventral splanchnic branches, which supply the gut and its derivatives. With the fusion of the dorsal aorta, the ventral branches fuse and form a series of unpaired segmental vessels, which run in the dorsal mesentery of gut and divide into ascending and descending branches. These vessels eventually form dorsal and ventral longitudinal anastomotic channels, With the formation of longitudinal anastomotic channels, numerous ventral splanchnic branches are withdrawn and only three trunks persist as coeliac artery for foregut, superior mesenteric artery to midgut, and inferior mesenteric artery to hindgut ${ }^{(3)}$.

\section{Case Report}

During routine dissection of posterior abdominal wall for undergraduate students, in a (45)fourty five year old embalmed male cadaver in the department of Anatomy. The following variation of celiac trunk was seen-The celiac trunk arose from the ventral surface of the abdominal aorta at the level of the intervertebral disc betweenT12 and L1vertebra. Coeliac trunk gave off left gastric artery, splenic artery and common hepatic artery. Common hepatic artery gave off Gastroduodenalartery, which is giving right gastric arteryand continued as Hepatic artery, which gave off right and left hepatic branches to the corresponding lobes of liver and further continued as accessory hepatic artery, which gave off Cystic artery to Gallbladder and another accessory hepatic artery divided into two small twigs to the right lobe of Liver. Left gastric artery gave off left inferior phrenic artery to the left dome of Diaphragm and another accessory hepatic artery which run in the fissure for ligamentumvenosum. In total three Accessory hepatic arteries were observed "Fig.1".

- One arising from left gastric artery,

- $\quad$ Another arising distal to the origin of hepatic artery, which also gave off Cystic artery.

- $\quad$ One arising from the above mentioned accessory hepatic artery to right lobe of Liver.

The Gallbladder also showed multiple stones black "Fig. 2" in colourwhichwere fused with one another. Gallbladder was adhered to the inferior surface ofLiver and Portahepatis. Gallstone inflammation is one of the causes of this adhesive scarring that binds tissues together.The reason for this is that membranes surrounding the gallbladder and helping it to hold it in place are formed of many layers, connective tissue layers are arranged in a regular pattern resembling woven wicker. If the wicker latticework is damaged, such as by inflammation or surgery the cells that regrow can be distorted. These distorted cells irregularly shaped and with 
weaker structural patterns form the scar tissue known as adhesions. Gallbladder adhesions do not cause any pain or symptoms but the conditions leading to the production of adhesions such as gallstones and inflammation can be extremely painful.

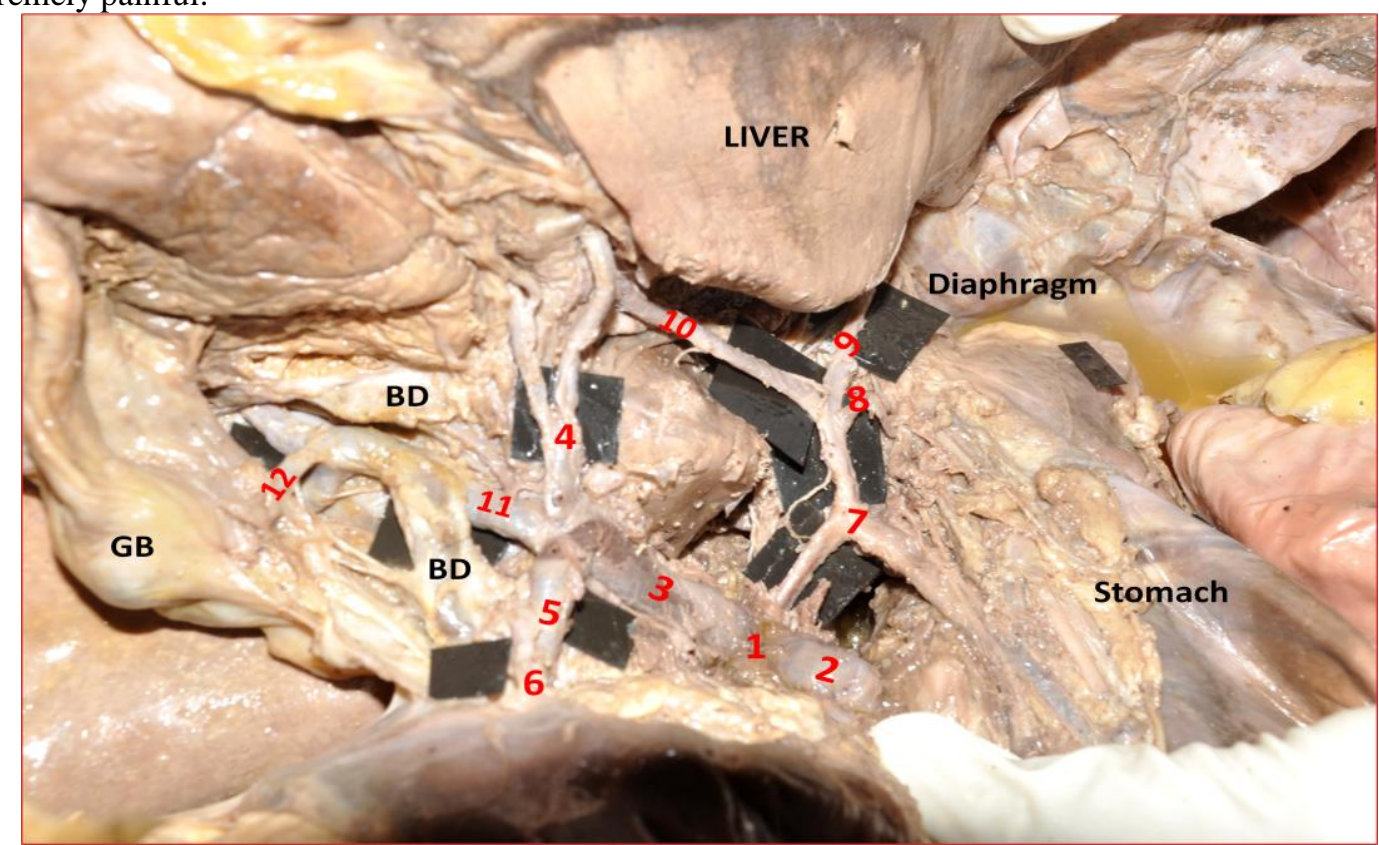

FIGURE-1.Dissection of Abdomen showing the variant branching pattern of Coeliac trunk.

1.Coeliac trunk,2.Splenic artery, 3.Common hepatic artery, 4.Hepatic artery, 5.Gastroduodenal artery, 6.Right gastric artery, 7.Left gastric artery,8.Oesophagial branch,9.Left inferior phrenic artery, 10.Accessory hepatic artery, 11.Accessory hepatic artery,12.Cystic artery,BD- Bile duct, GB- Gallbladder with Stones \& Adhesions.
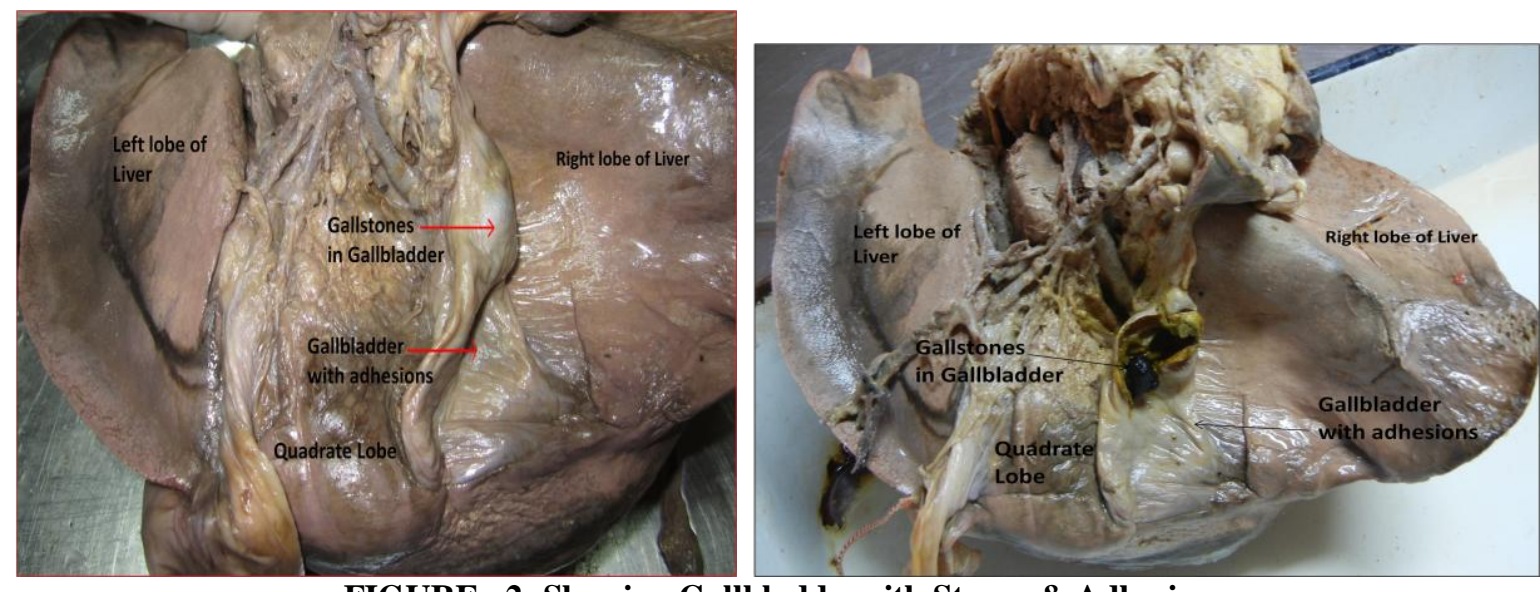

FIGURE- 2: Showing Gallbladderwith Stones \& Adhesions.

\section{Discussion}

Coeliac trunk is a chief artery of the fore gut. It supplies all derivatives of the foregut that lie in the abdominal cavity. It is about $1.25 \mathrm{~cm}$ in length and $6-8 \mathrm{~mm}$ in diameter. It gives of three main branches as left gastric, hepatic and splenic artery.

M buru et al ${ }^{(4)}$ did a study of 123 specimens at Kenyatta National hospital, Naieobi, Kenya and observed that the Coeliac trunk was trifurcated in $61.7 \%$, bifurcated in $17.9 \%$ and gave collateral branches in $20.3 \%$. Generally additional branches of Coeliac trunk other than the normalbranchesare referred to as collaterals. Collaterals observed included dorsal pancreatic, Gastroduodenal, inferior phrenic and ileal arteries. Dorsal pancreatic was the most common collateral occuring in $14.8 \%$ of cases, while inferior phrenic was found in $4.9 \%$, in our case inferior phrenic was seen arising as a collateral (branch of left gastric).

Piano et $\mathrm{al}^{(5)}$ summarizedthe origins of inferior phrenic artery as follows from Aorta(61.6\%), from Coeliacomesenteric system of aorta including the celiac trunk(28.2\%), and left gastric artery (2.9\%). The later visceral arteries (Adreno renal system of aorta) including the middle adrenal artery $(2.9 \%)$ and renal artery $(4.3 \%)$.We also reported origin of inferior phrenic from left gastric artery. 
Chiang et $\mathrm{al}^{(6)}$ studied 405 patients angiographically for evaluation of hepatic artery variations while a single accessory hepatic artery was found in $28 \%$ of these cases more than two hepatic arteries were found in $2 \%$ cases and seventeen patterns were identified in this study, some of the important patterns were accessory hepatic artery being direct branch from coeliac, branch from common hepatic as in our case, left gastric as in our case.

D'souzaetal.reportedan additional hepatic branch given by left gastric artery, which entered the left lobe of Liver, In our case also additional hepatic branch arose from left gastric artery and passing in the fissure for ligamentumvenosum to left lobe of Liver.

Variations of the coeliac trunk involving the hepatic arteries must be carefully understand for anastamosing the proper arteries in liver transplant surgeries. Anatomical segmental resection is now widely practiced and is superior to wedge resection in the treatment of metastatic liver cancer. These variations of celiac trunk are also important during the surgeries involving stomach, Duodenum, Pancreas, and Extra hepatic biliary apparatus.

\section{Developmental Correlation}

Anatomic variations of the celiac trunk are due to developmental changes in the ventral segmental (splanchnic) arteries. These ventral segmental arteries supply the yolksac, allantois and chorion. During embryological period there are longitudinal anastomoses between roots of upper four ventral segmental arteries of abdominal region. The two central roots disappear and the longitudinal anastomoses forms first $\&$ fourth root. The hepatic, Splenic and left gasticarteriesoriginate at this longitudinal anastomoses.These branches usually become separated from the fourth root (future superior mesenteric artery) below their last end. If this separation takes place at the higher level one of the branches is displaced to the superior mesenteric artery. If the first or the fourth root disappears a coeliacomesentric trunk will be formed ${ }^{(7)}$. In our case the variation of the coeliac trunk are due to the developmental changes in the longitudinal anastomoses between the above mentioned ventral segmental arteries.

\section{Conclusion}

Anatomical variations in the branchingpattern of coeliac trunk are of considerable importance in liver transplants, laparoscopic surgeries, radiological abdominal interventions and penetrating injuries to the abdomen. Vascular variations are usually asymptomatic. They may become important in patients undergoing Coeliacography for gastrointestinal bleeding, chemoembolization of pancreatic and liver tumors.Carefulidentification and dissection of coeliac trunk branches is therefore essential to avoid iatrogenic injury and useful in surgical, oncologic or interventional procedures and should be kept in mind to avoid complications.

\section{References}

[1]. StandringS. Gray's Anatomy, Anatomical basis of clinical practice, $40^{\text {th }}$ edition, Elsevier Churchill Livingstone, NewYork,2008:1073.

[2]. Halleri.A.IconAnatomicaeinquibuspraecipaepartescorporishumanidelineateproonunturetarteriarumpotssimumhistoriacontinetur.Gotting enVandenhoec.1756: VIII 270.

[3]. Datta A.K. Essentials of human Embryology( $5^{\text {th }}$ edition), Current books international, Kolkata.

[4]. M.buru KS, Alexander OJ, Hassan S, Bernard N.Variations in the branching pattern of the Coeliac trunk, a Kenyan Population. International Journal of Morphol2010, 28(1):199-204.

[5]. PianoDX, Ohtsuka A, Murakami.T. Typology of abdominal arteries, withspecialreferencestoinferior phrenic arteries and their oesophagealbranches. Acta Med Okayama,1988: 189 -96.

[6]. Chiang K, Chang P, Lee P, Ling C, Lee W, Lee C, ChouS Angiographic evaluation of hepatic artery variations in 405 cases.Chin J Radiol 2005:75 - 81.

[7]. Moore.K.L.Persaud TVN. The developing human (Clinically oriented embryology). $7^{\text {th }}$ edition, Philadelphia, Saunder $2003: 335$. 